more accurately assess the amount of antibody that may be transferred to the neonate and may, in turn, allow better prediction of severity of HDFN. ${ }^{3}$

This study assessed an ELISA-based commercial kit to see if it could accurately capture polyclonal anti-D from donor samples but found it had been developed to target only the first extracellular loop of the $\mathrm{RhD}$ protein and therefore, would be unsuitable for use in quantification of anti-D in patient samples.

References

1. Hirvonen M, Tervonen S, Pirkola A, et al. An enzyme-linked immunosorbent assay for the quantitative determination of anti-D in plasma samples and immunoglobulin preparations. Vox Sang 1995; 69: 341-6.

2. Ashford PR, Love M, Payne SG. Effect of Dithiothreitol treatment of plasma on automated anti-D assay. Vox Sang 1985; 49: 121-5.

3. Lambin P, Ahaded A, Debbia M, et al. An enzyme-linked immunosorbent assay for the quantitation of IgG anti-D and IgG subclasses in the sera of alloimmunized patients. Transfusion 1998; 38: 252-61.

\section{TIME-OF-FLIGHT MASS SPECTROMETRY IN THE DIAGNOSIS OF HAEMOGLOBIN KENYA}

Jordyn Moore ${ }^{1}$, Darrell Wang ${ }^{2}$, Howard Potter ${ }^{2}$, Richard King ${ }^{1,2,3}$

${ }^{1}$ Specialist Biochemistry, Canterbury Health Laboratories, Christchurch, New Zealand; ${ }^{2}$ Molecular Pathology, Canterbury Health Laboratories, Christchurch, New Zealand; and ${ }^{3}$ Department of Pathology, University of Otago, Christchurch, New Zealand

Haemoglobin $(\mathrm{Hb})$ variants are routinely detected by electrophoresis and high performance liquid chromatography (HPLC) in clinical laboratories. However, further molecular testing is often required to achieve a diagnosis. For rare variants molecular analysis may involve investigation of both the $\alpha$ - and $\beta$-globin gene clusters, as well as multiple techniques to detect point mutations and gene duplications or deletions. Time-of-flight (TOF) mass spectrometry is used as a secondary test in our laboratory to guide molecular analyses, saving both time and money. We present a case of Hb Kenya, a rare variant not typically seen in our patient population. $\mathrm{Hb}$ Kenya is associated with hereditary persistence of fetal haemoglobin (HPFH) and is the product of a $\gamma \beta$ fusion gene arising from abnormal crossing over during meiosis. This variant is not easily diagnosed by routine haematology or genetic methods and may be confused with other disorders. TOF mass spectrometry was used to identify the variant mass, enabling appropriate molecular testing to be designed for confirmation of the diagnosis. This case highlights the utility of TOF mass spectrometry for haemoglobinopathy and thalassaemia investigations.

\section{ATYPICAL PRESENTATION OF IGA-RELATED IMMEDIATE REACTION WITH BACK PAIN AND RELATIVE HYPOTENSION - ANAPHYLAXIS OR NOT?}

Eric Wenlong Li ${ }^{1}$, Pietro Di Ciaccio ${ }^{2}$, Kevin Fan ${ }^{1}$, John Taper ${ }^{1}$, Peta Dennington $^{3}$
${ }^{1}$ Department of Pathology, Nepean Hospital, NSW, Australia; ${ }^{2}$ Department of Haematology, Westmead and Nepean Hospitals, NSW, Australia; and ${ }^{3}$ Australian Red Cross Lifeblood, Alexandria, NSW, Australia

Anaphylactic transfusion reactions are rare occurring in approximately 1 in 50,000 red cell transfusions. ${ }^{1}$ One aetiology includes the development of antibodies against transfused IgA in patients with severe IgA deficiency. Diagnosis of IgA-related anaphylaxis involves the measurement of $\operatorname{IgA}$ levels and antiIgA antibodies. ${ }^{2}$ Recently, the evidence underlying this entity and the causal relationship between laboratory tests and clinical manifestation has been questioned. ${ }^{3}$ We describe a case of a 50year-old man with chronic anaemia with an infected enterocutaneous fistula. He received six red cell transfusions where he developed severe back pain and relative hypotension within 15 to 30 minutes of transfusion, settling after transfusion was ceased. He was afebrile with no respiratory, dermatological or gastrointestinal manifestations typical of anaphylaxis. Direct antiglobulin test was negative, and blood cultures were sterile. Tryptase taken 2.6 hours after symptom onset was raised $16 \mu \mathrm{g} / \mathrm{L}$ $(0.0-11.4 \mu \mathrm{g} / \mathrm{L})$. IgA level was $<0.10 \mathrm{~g} / \mathrm{L}(0.80-4.40 \mathrm{~g} / \mathrm{L})$. AntiIgA titre was $400 \mathrm{U} / \mathrm{mL}(0-7 \mathrm{U} / \mathrm{mL})$. IgA deficient components were prepared pre-operatively. He subsequently received three washed red cells uneventfully, one during a laparotomy for the enterocutaneous fistula.

This case describes an atypical presentation of a rare but important complication of blood transfusion, as well as the diagnostic process and subsequent management.

References

1. Sandler SG, Vassallo RR. Anaphylactic transfusion reactions. Transfusion 2011; 51: 2265-6.

2. Tinegate H, Birchall J, Gray A, et al. Guideline on the investigation and management of acute transfusion reactions Prepared by the BCSH Blood Transfusion Task Force. $\mathrm{Br} \mathrm{J}$ Haematol 2012; 159: 143-53.

3. Sandler SG, Eder AF, Goldman M, et al. The entity of immunoglobulin A-related anaphylactic transfusion reactions is not evidence based. Transfusion 2015; 55: 199-204.

\section{AUDIT OF BONE MARROW BIOPSY REPORTS SUBMITTED TO A MYELODYSPLASTIC SYNDROME REGISTRY}

Marsali Maclean, Linda Saravanan and on behalf of the MDSLink Steering Committee

Department of Haematology, Melbourne Pathology, Melbourne, Vic, Australia

Background: Reviewing a diagnosis of myelodysplastic syndrome (MDS) is challenging due to variability in morphological description and report content. Improved concordance in diagnosis may be achieved by adhering to morphological requirements based upon WHO classification criteria, ${ }^{1}$ and by following structured reporting guidelines.

Aim: We reviewed bone marrow biopsy reports submitted to a pilot Myelodysplastic Syndromes Registry (MDSLink) to confirm accuracy of diagnoses based on morphological description, according to WHO 2016 criteria. ${ }^{1}$ We also audited report content according to the RCPA Bone Marrow Specimen Structured Reporting Protocol. ${ }^{2}$ 